Rev. Elev. Méd. vét. Pays trop., 1977, 30 (3) : 309-314.

\title{
Note sur un essai de production de jeunes bovins précoces au Cameroun
}

\author{
par P. LHOSTE (*)
}

\begin{abstract}
RÉSUMÉ
L'auteur montre qu'il est possible d'obtenır, en Adamaoua, à partir de jeunes bovins de race locale et de métıs Charolais, des carcasses commercialement intéressantes, grâce à une finition précoce d'environ cinq mois après le sevrage. Les animaux ont été abattus à l'ảge de 13-14 mois et les poids moyens des carcasses obtenues sont de $151 \mathrm{~kg}$ en race locale et de $172 \mathrm{~kg}$ chez les métis taurins. Ces résultats confirment l'intérêt de la race locale plus rustique, pour l'intensification.
\end{abstract}

\section{INTRODUCTION}

Dans le cadre d'une expérimentation pluriannuelle de l'embouche bovine, menée au Cameroun, nous nous sommes intéressés à différents schémas de production de viande; comme nous l'avons rapporté par ailleurs, les conditions d'alimentation (1), ainsi que les types d'animaux ont varié : boeufs subadultes (1), jeunes mâles avant 2 ans (2). Dans l'essai rapporté ci-après, nous nous sommes intéressés à la production pour la boucherie d'animaux très jeunes (13-14 mois), de type «jeunes bovins précoces». Cet essai visait, en particulier, à la valorisation rapide de veaux métis zébu $\times$ taurin nés dans le cadre d'un programme d'insémination artificielle (3), mais dont l'élevage n'était pas prévu. A ces veaux métis taurins ont été associés des veaux zébus de race locale, à titre de témoins. Tous les animaux considérés ont été menés extensivement jusqu'à l'âge de 8 mois, correspondant au sevrage dans nos conditions d'élevage. Au cours d'une deuxième période, plus intensive, nous avons essayé de les préparer pour la bou-

(*) Adresse actuelle: Centre de Recherches Zootechniques B. P. 1152 Bouaké République de Côted'Ivoire. cherie en cinq mois, environ; cette note traite de cette période de préparation pour la boucherie à partir de veaux provenant des herbages.

\section{MATERIEL ET METHODES}

\section{Animaux}

L'essai est mené sur 21 jeunes bovins (8-9 mois en début d'expérience) répartis en deux groupes génétiques comme suit :

- 10 veaux mâles de race locale : zébu Foulbés de l'Adamaoua (photo $\mathrm{n}^{\circ} 1$ );

-11 veaux métis Charolais $\times$ zébu, répartis entre les deux sexes et de niveau de sang variable : soit 1/4 Charolais (photo $\mathrm{n}^{\mathrm{v}} 2$ ), soit $3 / 4$ Charolais (photo $n^{0} 3$ ) ; en effet, dans ce groupe, tous les veaux sont nés de mère demi-sang zébu $\times$ Charolais, mais l'ascendance paternelle est soit Charolaise $(\rightarrow 3 / 4$ Charolais) soit zébu ( $\rightarrow 1 / 4$ Charolais). Les effectifs considérés sont insuffisants pour tenir compte, dans ce groupe, des facteurs génétique et sexe. Nous comparerons donc globalement les performances de ce groupe des "métistaurins » à celles des zébus de race locale. 


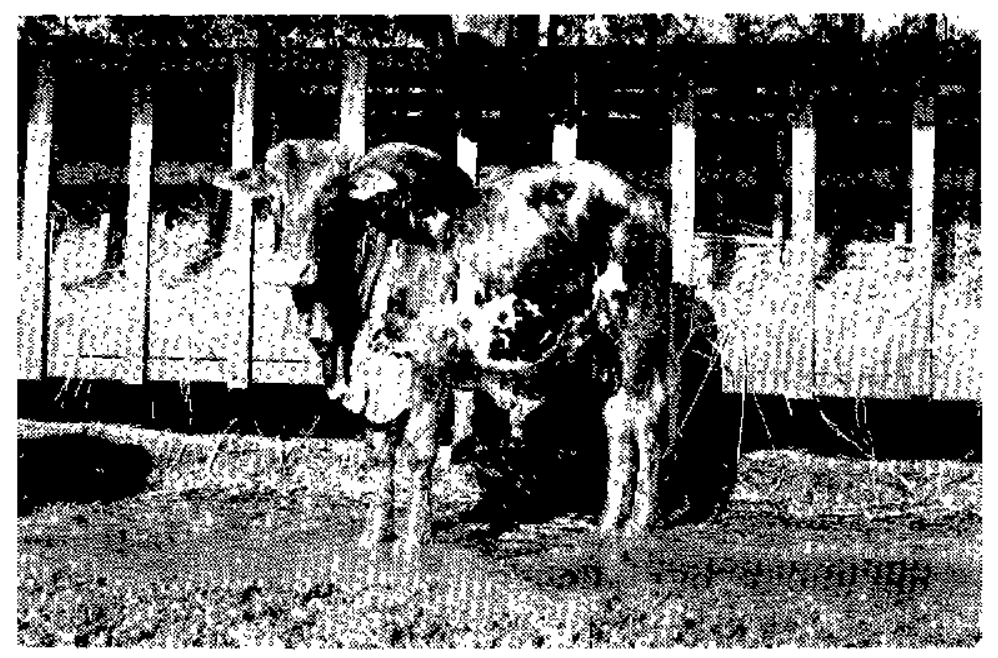

Photo $n^{\circ} 1$.

Taurillon de race locale Zébu Foulbé de l'Adamaoua.
Photo $\mathrm{n}^{\circ} 2$

Produit 1/4 Charolais, 3/4 Zébu.

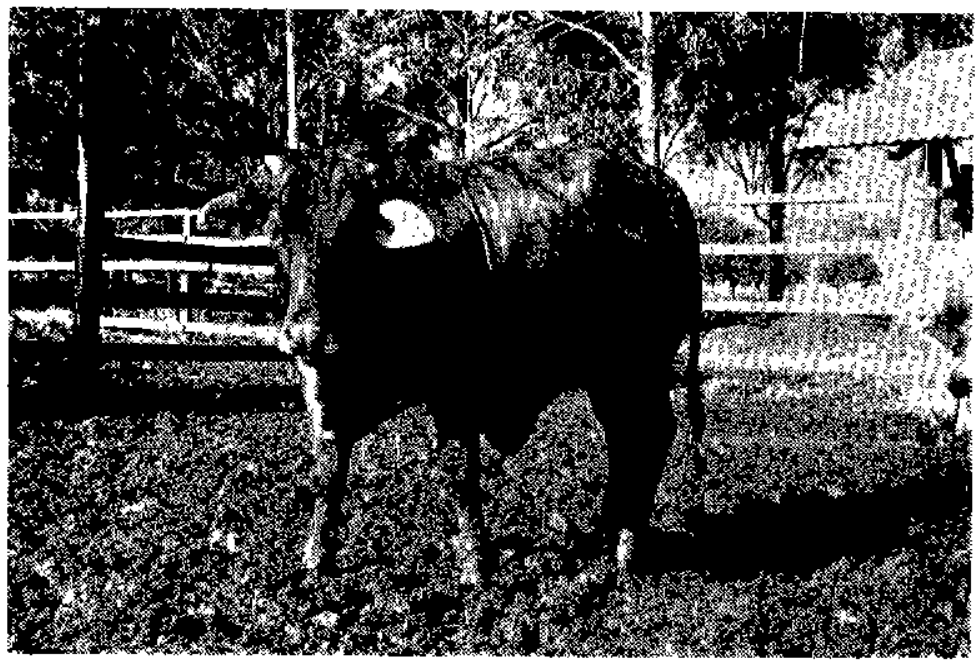

Photo $n^{\circ} 3$.

Produit 3/4 Charolais, 1/4 Zébu. 
Photo $n^{\circ} 4$.

Vue du lot d'embouche.

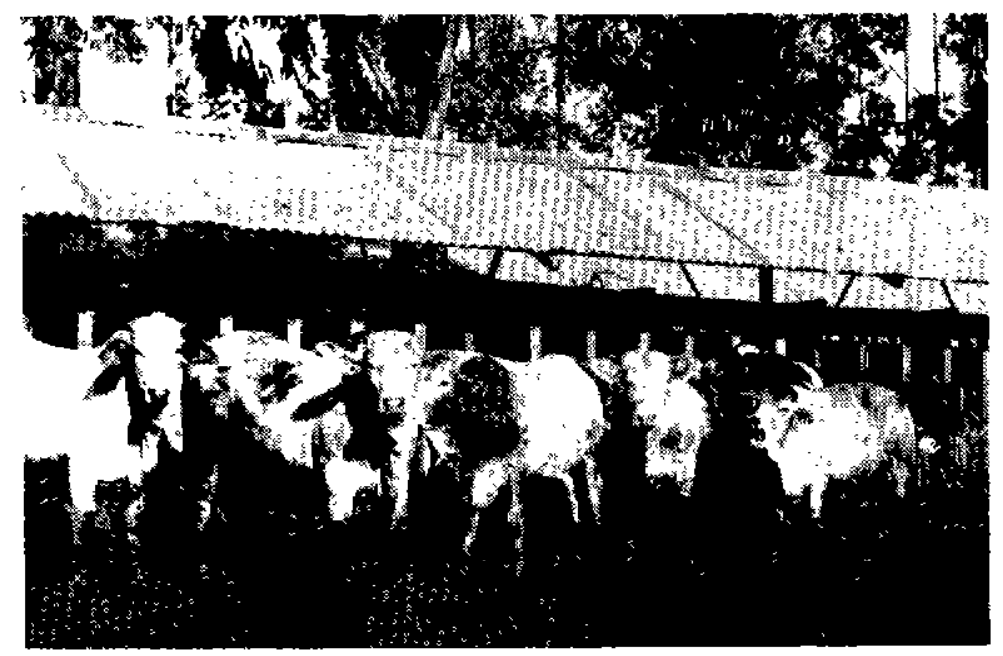

Alimentation et mode d'entretien (photo $\left.n^{\circ} 4\right)$

La ration, très simple, est composée de foin et d'un aliment concentré ; les quantités distribuées de fourrage et de concentré sont limitées pour permettre une croissance théorique d'environ $1 \mathrm{~kg} /$ jour/tête. Le foin utilisé provient d'une prairie améliorée à base de Brachiaria brizantha; l'aliment concentré est un granulé fabriqué à la station à partir de céréales et de sous-produits disponibles localement, suivant la formulation :

- Maïs : 50 p. 100

- Tourteau de graines de coton : 26 p. 100

- Farine de blanchiment de riz : 22 p. 100

- Minéraux

2 p. 100

Sur la base d'analyses bromatologiques effectuées par le laboratoire de nutrition de l'IEMVT, la valeur nutritive de cet aliment a pu être évaluée à :

1 U. F. $/ \mathrm{kg}$ et $150 \mathrm{~g}$ de M. A. D. $/ \mathrm{kg}$.

Les animaux sont entretenus pendant toute la durée de l'essai dans des parcs d'embouche où ils disposent en permanence d'eau et d'un mélange minéral sous forme de pierre à lécher.

\section{Déroulement de l'essai et observations}

L'essai a débuté vers le milieu de la saison sèche, début février, après une phase d'adaptation d'environ deux semaines au cours de laquelle les animaux s'habituent aux nouvelles conditions d'entretien et d'alimentation. Tous les animaux sont pesés toutes les semaines, le matin à jour et heure fixes ; de plus, après chaque phase expérimentale de 4 semaines, des «pesées de référence »sont effectuées 3 jours consécutifs dans les mêmes conditions. Le dépouillement de l'essai a été effectué sur la base da ces pesées de référence.

L'essai a duré environ cinq mois, et tous les animaux ont été abattus à son terme.

\section{RESULTATS ET DISCUSSION}

\section{Comportement des animaux}

L'essai s'est déroulé comme prévu, de février à juin, c'est-à-dire en fin de saison sèche et début de saison des pluies. Les animaux se sont très rapidement adaptés à ce régime nouveau, et l'installation des pluies vers le milieu de l'expérience n'a pas entraîné de sérieuses perturbations. Deux animaux du groupe des métis Charolais ont dû être abattus, l'un à la suite d'un accident (fracture), et l'autre pour des raisons sanitaires; il s'agissait de deux 3/4 Charolais, ce qui confirme la plus grande fragilité, observée par ailleurs, des animaux de ce groupe génétique par rapport aux $3 / 4$ zébus et zébus purs de race locale.

L'essai a été quelque peu écourté en raison du risque que présentait une épizootie de fièvre aphteuse qui sévissait à cette époque en Adamaoua ; cet événement nous a incité à abattre les animaux de l'essai plus tôt que prévu. 
Evolution pondërale moyenne pour les 2 lcts avec gains journaliers moyens ( $g / j$ ) par phase

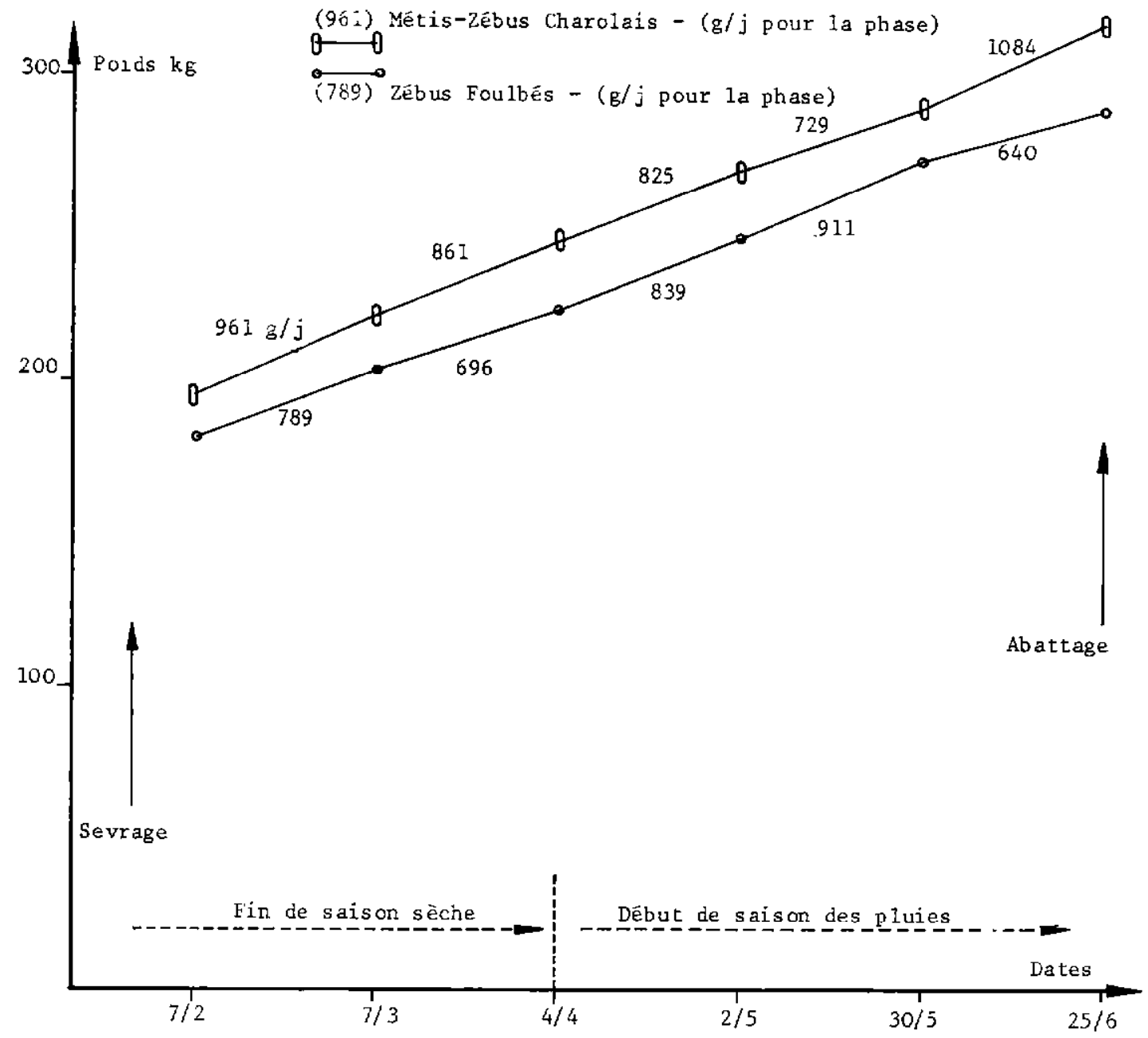

Résultats : croissance, conversion et rendement boucher

Nous présentons au graphique ci-dessus l'évolution pondérale moyenne enregistrée pour les deux lots de l'essai. Pour des croissances moyennes très voisines de $900 \mathrm{~g} / \mathrm{j}$ chez les métis taurins et de $800 \mathrm{~g} / \mathrm{j}$ chez les zébus, nous remarquons l'allure très régulière des courbes de croissance qui s'ajustent aisément sur des droites pour les cinq phases considérées. Il n'y a donc pas de ralentissement de la croissance pondérale au cours de cet essai, contrairement à ce que nous avons établi précédemment $(1,2)$, et ce fait particulier s'explique ainsi :

a) les animaux de l'essai considéré sont très jeunes et ils extériorisent une croissance plus régulière et très soutenue,

b) les rations distribuées sont limitées pendant tout l'essai et elles ne permettent pas aux animaux d'extérioriser une croissance maximale.

L'influence du facteur phase expérimentale n'est pas significative et nous présentons au tableau ci-après les résultats moyens établis par groupe pour l'ensemble de l'essai :

croissance pondérale $=$ poids en $\mathrm{kg}$

et gain de poids journalier moyen $\mathrm{g} / \mathrm{j}$

conversion de la ration $=$ indice de consommation en U. F. $/ \mathrm{kg}$

résultats d'abattage $=\left\{\begin{array}{l}\text { poids de la carcasse }(\mathrm{kg}) \\ \text { rendement brut (p. 100) }\end{array}\right.$ 
Résultats moyens pour l'ensemble de l'essai.

\begin{tabular}{|c|c|c|}
\hline & $\begin{array}{c}9 \text { Métis } \\
\text { Zébu x Charolais }\end{array}$ & $\begin{array}{l}10 \text { Zëbus } \\
\text { de race locale }\end{array}$ \\
\hline $\begin{array}{l}\text { Données pondérales : } \\
\text { - Poids moyen au départ (kg) } \\
\text { - Poids moyen à la fin (kg) } \\
\text { - Gain de poids journalier moyen } \\
\quad(\mathrm{g} / \mathrm{j}) \\
\text { Indice de consommation } \\
\text { Resultats d'abattage : } \\
\text { - Poids de la carcasse (kg) } \\
\text { - Rendement brut (p.100) }\end{array}$ & $\begin{array}{l}194,4 \mathrm{~kg} \\
316 \mathrm{~kg} \\
889 \mathrm{~g} / \mathrm{j} \\
6,6 \mathrm{U} . \mathrm{F} . / \mathrm{kg} \\
172 \mathrm{~kg} \\
54,4\end{array}$ & $\begin{array}{l}181,3 \mathrm{~kg} \\
287,9 \mathrm{~kg} \\
778 \mathrm{~g} / \mathrm{j} \\
7,5 \text { U.F. } / \mathrm{kg} \\
151 \mathrm{~kg} \\
52,4\end{array}$ \\
\hline
\end{tabular}

Les consommations moyennes d'aliment ont évolué au cours de l'essai en fonction de l'augmentation du poids vif; elles s'établissent en moyenne à $4,8 \mathrm{~kg} / \mathrm{j} /$ tête de concentré et $2,3 \mathrm{~kg}$ de foin. Les indices de consommation calculés sont satisfaisants et, comme les gains de poids, ils sont plus favorables chez les métis-taurins que chez les zébus.

Au niveau des résultats d'abattage, nous observons que les carcasses obtenues avec ces très jeunes animaux (13-14 mois) sont d'un poids commercial intéressant, supérieur à $150 \mathrm{~kg}$.

Nous observons sur la plupart des critères étudiés une supériorité des animaux métis-taurins sur les zébus : gains de poids, indice de consommation, rendement à l'abattage. Toutefois, ces différences restent assez faibles ( +14 p. 100 sur la vitesse de croissance et le poids à la carcasse), et ceci nous confirme les aptitudes de la race locale des zébus Foulbés de l'Adamaoua pour lintensification. Dans les conditions où nous avons opéré, l'opération a pu être équilibrée économiquement.

Ce type de production d'animaux très jeunes peut paraître surprenant dans un pays où les fourrages sont très abondants; il s'agit, en effet, d'un schéma extrême qui n'est pas adapté à toutes les situations; toutefois, pour certains contextes particuliers, où on dispose de sousproduits bon marché et où on a intérêt à faire tourner les animaux rapidement, ce type de solution peut être avantageux. Une source d'économie importante, en effet, est due au fait qu'il n'y a pas à assurer plusieurs années de suite les charges d'élevage et les risques de saison sèche (compléments, pertes,...), car les veaux peuvent être introduits dans l'atelier de finition dès leur sevrage.

\section{CONCLUSION}

Les résultats obtenus dans ce bref essai complètent donc notre connaissance des possibilités de finition pour la viande des bovins de l'Adamaoua. Chez de très jeunes animaux de type «Jeunes Bovins Précoces », nous avons pu obtenir, en 5 mois d'embouche à partir du sevrage, des carcasses commercialement intéressantes pour la région : $151 \mathrm{~kg}$ de moyenne en race locale et $172 \mathrm{~kg}$ chez les métis-taurins.

Nous observons une fois de plus l'amélioration des performances obtenues par le croisement, tant pour la vitesse de croissance que pour l'efficacité de la ration et le poids de la carcasse ; la race locale (zébu Foulbé de l'Adamaoua), nettement supérieure en rusticité, présente, cependant, une bonne adaptation à ce type de production. Les possibilités d'exploitation précoce des bovins ne sont pas à exclure dans des conditions particulières qui peuvent apparaître au Cameroun : nous pensons, en particulier à des ateliers d'embouche intensive qui pourront être associés à des projets agroindustriels (sucreries, huileries, rizeries), où une partie importante de la ration des animaux peut être fournie à bon marché. 


\section{SUMMARY}

\section{Note about an experiment of early young cattle production in Cameroon}

With Adamawa breed and Charolais crossbred cattle, it is possible to obtain in Cameroon, at 13-14 months of age, carcasses of rather good commercial value. After weaning at 8 months, early fattening is run during about 5 months to achieve an average of $151 \mathrm{~kg}$ carcass weight with local cattle and $172 \mathrm{~kg}$ with crossbred. These results show the interest of local breed for intensive production.

\section{RESUMEN}

Nota sobre un ensayo de producción de novillos precoces en Camerún

El autor muestra que se puede obtener en Adamaua, a partir de novillos de raza del pais y de mestizos Charolais, canales comercialmente interesantes, gracias a un acabado precoz de unos cinco meses después del destete. Se mataron los animales a 13-14 meses de edad. Los pesos medios de los canales obtenidos son de $151 \mathrm{~kg}$ para la raza del pais y de $172 \mathrm{~kg}$ para los mestizos taurinos. Dichos resultados confirman el interes de la raza del pais, más, rústica, para la intensificación.

\section{BIBLIOGRAPHIE}

1. LHOSTE (Ph.), DUMAS (R.). Embouche intensive des zébus de l'Adamaoua. I. Comparaison de différents systèmes d'alimentation (1970). II. Influence de la durée de la période d'embouche (1971). Rev. Elev. Méd. vét. Pays trop., 1972, 25 (2) : 259-293.

2. LHOSTE (Ph.), PIERSON (J.). Embouche intensive de jeunes mâles : comparaison de taurillons et bou- villons de trois races. Colloque Embouche, Dakar, déc. 1973, Actes pp. 233-236.

3. LHOSTE (Ph.), PIERSON (J.). L'expérimentation de l'insémination artificielle au Cameroun par importation de semence congelée. I. Insémination artificielle de femelles zébus en chaleurs naturellement. Rev. Elev. Méd. vét. Pays trop., 1975, 28 (4) : 513-522. 